\title{
Ischemic Conditioning-Mediated Myocardial Protection in Relation to Duration of Coronary Occlusion
}

\author{
John G. Kingma Jr. \\ Department of Medicine, Faculty of Medicine, Pavillon Ferdinand-Vandry, Université Laval, Québec, Canada \\ Email: john.kingma@fmed.ulaval.ca
}

How to cite this paper: Kingma Jr., J.G. (2021) Ischemic Conditioning-Mediated Myocardial Protection in Relation to Duration of Coronary Occlusion. World Journal of Cardiovascular Diseases, 11, 210-222.

https://doi.org/10.4236/wjcd.2021.113021

Received: February 23, 2021

Accepted: March 27, 2021

Published: March 30, 2021

Copyright $\odot 2021$ by author(s) and Scientific Research Publishing Inc. This work is licensed under the Creative Commons Attribution International License (CC BY 4.0).

http://creativecommons.org/licenses/by/4.0/

\begin{abstract}
Background: Myocardial ischemia is a dynamic process whereby a cascade of events is initiated to stimulate transition from reversible to irreversible cellular injury. Non-pharmacologic approaches to cellular protection, such as ischemic conditioning, delay onset of cellular injury in most organs in a host of animal species; however the degree of protection is limited to rather short durations of ischemia. In the present study, we examined whether protection afforded by ischemic conditioning could be extended beyond currently established limits of coronary occlusion in an in situ animal model. Methods: Rabbits $(\mathrm{n}=106)$ were exposed to 30-, 60-, 120-, 180-, 240-, or 360-min coronary occlusion followed by 180 -min coronary reperfusion (i.e. non-conditioned control groups). Ischemic conditioned rabbits were pre-treated by ischemic conditioning (i.e. 2-cycles of 5-min coronary occlusion and 5-min reperfusion) prior to a prolonged period of ischemia as described above. Area at risk (AR; by fluorescent microparticles) and area of necrosis (AN; by tetrazolium staining) were quantified by planimetry. Serum troponin I levels were assessed at baseline (i.e. before experimental protocol) and at the end of the experiment. Results: Changes in heart rate and hemodyamic indices were similar for all groups regardless of duration of ischemia and regardless of treatment (i.e. non-conditioned vs. ischemic conditioned). Infarcts (as percent AR) were markedly smaller ( $35 \%)$ in ischemic conditioned rabbits (vs. controls) for the 30 -min coronary occlusion group. With longer durations of coronary occlusion (60-, 120-, 180-, 240-min) infarcts were smaller ( 20\%) in ischemic conditioned groups but protection afforded was not statistically significant. With 360-min coronary occlusion, infarct size was the same for both treatment groups. Serum troponin I levels were greater in relation to infarct size as expected but no differences were detected between treatments regardless of ischemic duration. Conclusions: Ischemic conditioning limits infarct development; how-
\end{abstract}


ever, protection is limited when the duration of ischemia is extended beyond 4 hours. These findings provide further support for the concept that ischemic conditioning can delay, but does not limit myocyte necrosis. Underlying mechanisms for cellular protection remain to be established.

\section{Keywords}

Ischemia, Reperfusion, Infarct Size, Biomarkers, Ischemic Conditioning, Rabbits

\section{Introduction}

Abundant scientific reports have documented that exposure to brief and repetitive episodes of acute vascular occlusion with intermittent reperfusion prior to a prolonged period of sustained vessel occlusion- "ischemic conditioning"-can significantly reduce the extent of organ injury [1] [2] in animals [3] and humans [4] [5] as long as there is adequate reperfusion of the ischemic zone.

In the heart, "ischemic conditioning" also reduces the incidence of ventricular dysrhythmias and post-ischemic contractile dysfunction (i.e. myocardial stunning). Protection afforded by this non-pharmacologic intervention is immediate and can be triggered by release of endogenous mediators (nitric oxide, adenosine, bradykinin) [6] [7] and activation of complex second messenger systems [8] and antioxidant pathways [9]. A "second window (delayed)" of protection which depends on induction of cytoprotective proteins has also been described [10] [11]. While protection against ischemic injury with this non-pharmacologic intervention has been documented in most organs studied [12] [13] its clinical usefulness is somewhat limited. Current studies, particularly in humans, are focused on the protection afforded by an additional paradigm of the phenomenon defined as "remote" or "distance" conditioning [14] [15] [16].

The goal of the present study was to investigate whether ischemic conditioning cytoprotection is possible with extended durations of coronary occlusion in an in situ animal model with sparse native collateral circulation.

\section{Materials and Methods}

Animals used in these studies were maintained in accordance with the policies and guidelines of the Canadian Council on Animal Care. The institutional animal care committee at Laval University approved experimental and surgical protocols.

Animals: Pathogen-free, healthy New-Zealand White male rabbits $(2-3 \mathrm{Kg}$ body weight; 11 - 12 months of age) from Charles River Laboratories (Québec, Canada) were used. Animals were maintained in single cages under conditions of constant temperature and humidity and kept on a strict 12:12 h dark-light cycle; food (comprising $17.7 \%$ crude protein, $3.3 \%$ fat and $13.7 \%$ crude fiber; (Harlan Laboratories Inc., Montreal, QC) and water were accessible ad libitum. 
Surgical and experimental procedures. Animals were pre-medicated with acepromazine $(0.5 \mathrm{mg} / \mathrm{Kg}, \mathrm{IM}) ; 20$-min later they were anesthetized with pentobarbital sodium $(25 \mathrm{mg} / \mathrm{Kg}, \mathrm{IV})$. Body temperature was monitored during the entire experimental protocol with a rectal temperature probe; core temperature was maintained with warming pads. The trachea was intubated via a cervical cutdown; ventilation was ensured with a positive-pressure small animal ventilator (MDI Inc., Mobile, AL). The jugular vein was cannulated (PE 100) for withdrawal of blood-an initial sample was obtained prior to a left thoracotomy for determination of baseline troponin I levels.

After thoracotomy, the heart was exposed; a fluid-filled polyethylene cannula (PE-90) in the LV chamber (via the apex) connected to a Gould manometer measured LV developed pressure. A silk suture (3 - 0; Ethicon, Somerville, NJ) was placed underneath the anterolateral branch of the left main coronary artery midway between the base and apex; the end of the suture was drawn through a polypropylene tube to form a snare. At onset of coronary occlusion, ischemia was confirmed by regional epicardial cyanosis and ST segment elevation of the electrocardiogram. Coronary reperfusion of the infarct-related artery was achieved by releasing the snare and was confirmed by hyperemic blushing.

Hearts that developed ventricular fibrillation (VF) were successfully cardioverted by gentle flicking of the left ventricle (electrical cardioversion was not used). If cardioversion was not successful, the animal was excluded from the study. Lead II electrocardiogram and left ventricular pressure was recorded continuously using a 4-channel EasyGraf ${ }^{\mathrm{TM}}$ recorder (TA-240 Gould Instruments, Montreal, QC).

Experimental protocol. Rabbits were randomly assigned a treatment group; hearts in the ischemic conditioning group were exposed to two cycles of 5-min coronary occlusion plus 5-min reperfusion; in control animals (i.e. non-conditioned group) a 20-min wait period was allowed prior to onset of coronary occlusion. Ischemic durations were 30,60,120,180, 240, or 360-min; in all animals reperfusion lasted for $3 \mathrm{~h}$. Heparin or lidocaine were not administered because of their potential cytoprotective actions [17] [18].

At the end of reperfusion, but before sacrifice (intra-atrial injection of hypertonic $\mathrm{KCl}$ ), a venous blood sample was obtained for assessment of plasma troponin I. The heart was excised, attached to a Langendorff perfusion system and immediately perfused (65 $\mathrm{mmHg}$ pressure) for 1-min with warmed saline (to remove blood) followed by 2,3,5-triphenyl tetrazolium chloride $(2 \% \mathrm{w} / \mathrm{v})$ for 20 -min. At the end of this period, the coronary suture was re-tied and zinccadmium sulfide micro particles $(1-100 \mu \mathrm{m})$ were injected to delineate the anatomic risk zone. Hearts were then removed from the perfusion system and fixed in $10 \%$ formalin until analysis.

Postmortem analysis. The LV was sectioned into $2 \mathrm{~mm}$ slices parallel to the atrioventricular groove; the outline of each slice and tetrazolium negative (i.e. infarcted) regions were traced onto clear acetate sheets. Illumination, of micro 
particles within the ventricular slices, with an ultraviolet lamp allowed delineation (and tracing) of the anatomical risk zone. Total LV cross-sectional, risk and infarct areas were determined from enlarged tracings (1.5X); risk zone, infarct and LV volumes, for each slice were calculated as the sum of the area obtained by computerized planimetry (SigmaScan, SPSS, IL, USA) and ventricular slice thickness. Values from the sequential slices were summed to provide the total volume measure of LV, risk and infarct zones; infarct size was normalized to anatomical risk zone size.

Assessment of serum troponin I levels. Quantitative determination of cardiac troponin I was performed using a commercially available immunoenzymatic fluorescent kit (Stratus II, Dade International Inc., Miami, FL) with two monoclonal antibodies specific for independent epitopes of cTnI; analytical sensitivities were indicated as $0.35 \mathrm{ng} / \mathrm{mL}$ at the $95 \%$ confidence level. All samples were analyzed in duplicate within $2 \mathrm{~h}$ of collection. Blood samples were centrifuged, at $1000 \mathrm{rpm}$ for $10 \mathrm{~min}$, within 20 -min of collection and serum was stored on ice.

Data analysis. Data analysis was done using SAS (Statistical Analysis Systems; SAS Institute), a statistical software package for the personal computer. Cardiac hemodynamics and infarct size (expressed as percent of risk zone size) were analyzed by two-way analysis of variance (ANOVA) with Bonferroni post-hoc testing. Infarct size varies as a function of risk zone size [19]; differences between groups for infarct volume $\left(\mathrm{mm}^{3}\right)$ were evaluated by analysis of covariance (ANCOVA) with risk volume $\left(\mathrm{mm}^{3}\right)$ being the covariate-this relationship is considered to have a non-zero $\mathrm{x}$-intercept [20]. Linear regression analysis was done on pooled data for risk zone volume versus infarct volume, infarct size versus heart rate-systolic blood pressure product and infarct size versus plasma troponin I. The incidence of VF incidence was evaluated using the KruskalWallis test. Group sample size was calculated to provide a $\geq 0.90$ power to detect a minimum reduction of $15 \%$ (with an expected deviation of 8 ) of infarct size. All data are expressed as mean (SEM), and statistical significance is assumed for $\mathrm{p}<0.05$.

\section{Results}

The incidence of VF was 28\% (15 of 53) in non-conditioned (i.e. controls), and $21 \%$ (11 of 53) in ischemic conditioned animals ( $\mathrm{p}=$ NS between groups). Nonconvertible VF occurred in two rabbits that were excluded from the statistical analysis. This report is based on the 104 rabbits that survived the experimental protocol.

Cardiac hemodynamics. A summary of changes in heart rate-blood pressure product data throughout the experimental protocol for each group is shown in Table 1. Values for this index decreased relative to baseline in all groups but a significant group effect was not observed; these data are consistent with previous observations for ischemia-reperfusion in this animal model. 
Myocardial infarct size. Heart weight and mean LV area were similar for all groups; risk zone area was also comparable and comprised $\pm 45 \%$ of LV mass. Infarct size data for all experimental groups are shown Figure 1. Ischemic conditioning in rabbits exposed to 30-min coronary occlusion reduced infarct size (normalized to risk zone area) by approximately 35 percent. Infarct size was also less pronounced ( 20 percent) in ischemic conditioned rabbits that underwent 60-, 120-, 180- or 240-min coronary occlusion but did not achieve a level of statistical significance. No protection was observed in ischemic conditioned rabbits exposed to 360-min coronary occlusion. The regression relation (pooled data) between area of necrosis and area at risk was not significantly altered by ischemic conditioning as shown in Figure 2; larger infarcts are associated with larger risk regions.

Protection against ischemic injury in the 30 -min ischemic conditioning group was not due to significant lowering of myocardial oxygen demand (using the heart rate-blood pressure product index); the relationship between heart ratepressure product and infarct size is shown in Figure 3. Larger infarcts were observed in animals with a higher level of myocardial oxygen demand.

Table 1. Heart rate-blood pressure product data.

\begin{tabular}{|c|c|c|c|c|c|c|c|c|c|c|}
\hline \multirow{2}{*}{$\begin{array}{c}\text { Interventior } \\
\text { Minutes }\end{array}$} & \multicolumn{7}{|c|}{ Occlusion } & \multicolumn{3}{|c|}{ Reperfusion } \\
\hline & 0 & 30 & 60 & 120 & 180 & 240 & 360 & 30 & 120 & 180 \\
\hline \multicolumn{11}{|c|}{ Non-conditioned } \\
\hline 30 & $1.99 \pm 0.42$ & $1.78 \pm 0.45$ & & & & & & $1.71 \pm 0.26$ & $1.69 \pm 0.18$ & $1.61 \pm 0.30$ \\
\hline 60 & $1.99 \pm 0.46$ & $1.77 \pm 0.41$ & $1.78 \pm 0.44$ & & & & & $1.68 \pm 0.40$ & $1.64 \pm 0.37$ & $1.68 \pm 0.38$ \\
\hline 120 & $1.92 \pm 0.33$ & $1.86 \pm 0.47$ & $1.84 \pm 0.37$ & $1.81 \pm 0.53$ & & & & $1.88 \pm 0.53$ & $1.86 \pm 0.28$ & $1.74 \pm 0.55$ \\
\hline 180 & $2.14 \pm 0.31$ & $1.94 \pm 0.18$ & $1.85 \pm 0.14$ & $1.91 \pm 0.17$ & $1.77 \pm 0.18$ & & & $1.80 \pm 0.19$ & $1.79 \pm 0.37$ & $1.88 \pm 0.46$ \\
\hline 240 & $1.91 \pm 0.27$ & $1.65 \pm 0.34$ & $1.69 \pm 0.30$ & $1.62 \pm 0.35$ & $1.50 \pm 0.28$ & $1.54 \pm 0.35$ & & $1.61 \pm 0.37$ & $1.59 \pm 0.31$ & $1.57 \pm 0.28$ \\
\hline 360 & $2.11 \pm 0.43$ & $2.01 \pm 0.49$ & $2.12 \pm 0.44$ & $2.10 \pm 0.44$ & $2.15 \pm 0.45$ & $1.92 \pm 0.41$ & $1.95 \pm 0.46$ & $1.82 \pm 0.41$ & $1.71 \pm 0.39$ & $1.71 \pm 0.44$ \\
\hline \multicolumn{11}{|c|}{ Ischemic conditioning } \\
\hline 30 & $2.05 \pm 0.39$ & $1.78 \pm 0.31$ & & & & & & $1.86 \pm 0.29$ & $1.85 \pm 0.49$ & $1.81 \pm 0.37$ \\
\hline 60 & $1.75 \pm 0.25$ & $1.57 \pm 0.32$ & $1.62 \pm 0.24$ & & & & & $1.66 \pm 0.26$ & $1.53 \pm 0.24$ & $1.39 \pm 0.33$ \\
\hline 120 & $2.11 \pm 0.54$ & $1.92 \pm 0.50$ & $1.88 \pm 0.40$ & $1.88 \pm 0.50$ & & & & $1.81 \pm 0.44$ & $1.83 \pm 0.47$ & $1.79 \pm 0.35$ \\
\hline 180 & $2.22 \pm 0.47$ & $1.85 \pm 0.43$ & $1.94 \pm 0.47$ & $1.90 \pm 0.50$ & $1.91 \pm 0.51$ & & & $1.88 \pm 0.46$ & $1.91 \pm 0.55$ & $1.84 \pm 0.53$ \\
\hline 240 & $2.00 \pm 0.24$ & $1.77 \pm 0.19$ & $1.84 \pm 0.21$ & $1.82 \pm 0.35$ & $1.80 \pm 0.29$ & $1.88 \pm 0.39$ & & $1.79 \pm 0.41$ & $1.53 \pm 0.48$ & $1.51 \pm 0.43$ \\
\hline 360 & $1.85 \pm 0.32$ & $1.71 \pm 0.28$ & $1.92 \pm 0.31$ & $1.97 \pm 0.29$ & $1.98 \pm 0.30$ & $1.99 \pm 0.46$ & $1.82 \pm 0.39$ & $1.73 \pm 0.35$ & $1.57 \pm 0.48$ & $1.48 \pm 0.47$ \\
\hline
\end{tabular}

Data are mean $\pm 1 \mathrm{SD}$. Heart rate-blood pressure product data was analyzed by repeated-measures ANOVA; values decreased over time in all groups and no significant group effects were observed. Heart rate-blood pressure product was calculated by multiplying heart rate (beats/min) and systolic blood pressure $(\mathrm{mmHg})\left(\times 10^{4}\right)$. 


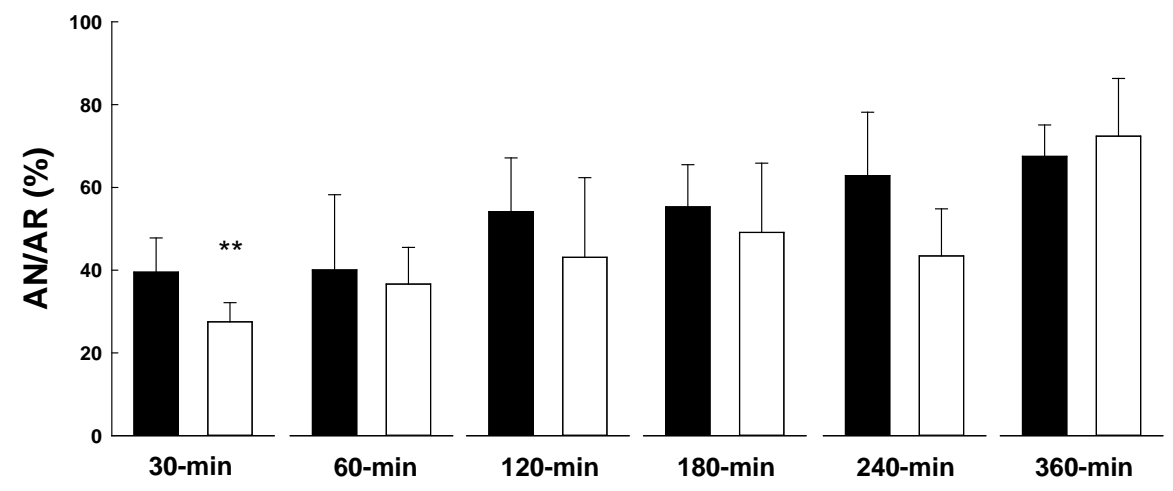

Figure 1. Infarct size (percent risk zone size) for non-conditioned (i.e. control; filled bars) and ischemic conditioned (open bars) groups exposed to different durations of ischemia (range: 30 - to $360-\mathrm{min}$ ). Results are means $\pm \mathrm{SD}$; ${ }^{*}$ denotes $P \leq 0.05$ determined by two-way ANOVA with Bonferroni post-hoc testing.

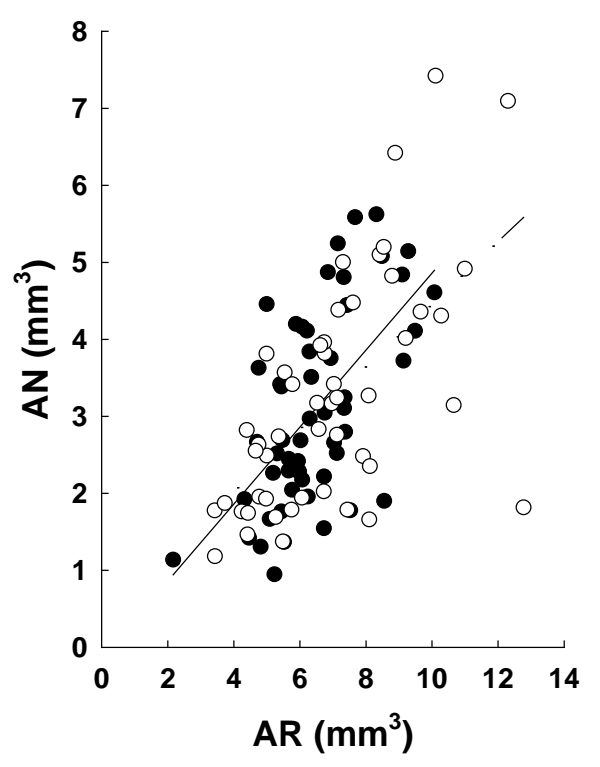

Figure 2. Scattergram showing the relation between necrosis (percent of LV area) plotted against area at risk (as percent of LV area) for non-conditioned (closed circles; solid line) and ischemic conditioned (open circles; dashed line) for all groups (pooled data). The extent of necrosis increases in relation to area at risk. Statistical differences were not observed between treatments (i.e. non conditioned: $\mathrm{Y}=-0.138+0.5 \mathrm{x} ; \mathrm{r}^{2}=0.35$ vs. ischemic conditioned: $\left.\mathrm{Y}=0.403+0.4 \mathrm{x} ; \mathrm{r}^{2}=0.38\right)$.

Serum troponin I levels. Cardiac troponin I was not detectable in blood samples obtained prior to coronary occlusion (i.e. baseline). Circulating troponin I levels increased (range: $10-100 \mu \mathrm{g} / \mathrm{L}$ ) in relation to the duration of coronary occlusion; however, statistical differences were not discerned for either non-conditioned or ischemic conditioned rabbits regardless of the duration of ischemia. A correlation between cardiac troponin I and infarct size is shown in Figure 4 ( $p=0.115$ between groups). Ischemic conditioning had no effect on the overall relation; however, greater release of troponin I is evident with larger infarcts (due to higher degree of cellular injury). 


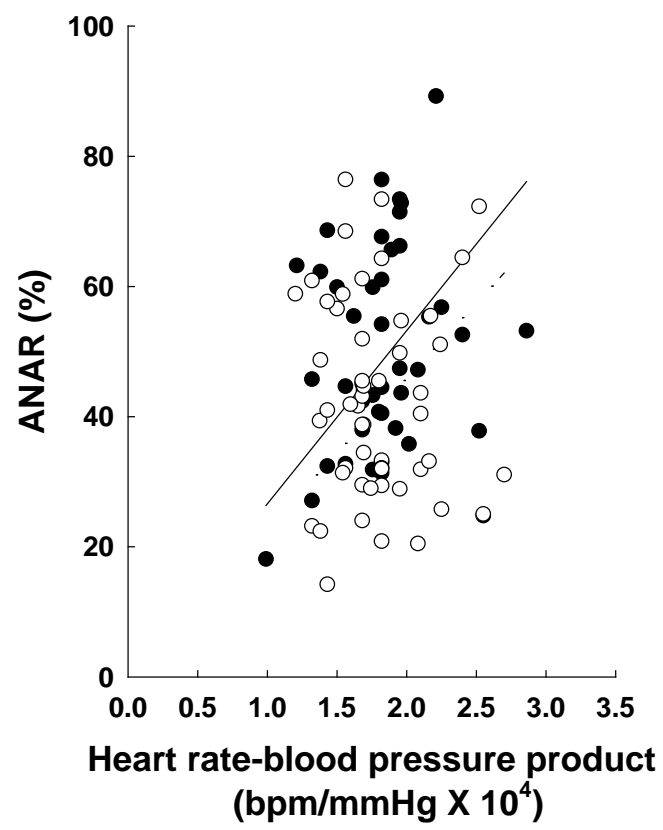

Figure 3. Scattergram showing relation between infarct size (as percent of risk zone size) and heart rate-blood pressure product for non-conditioned (closed circles; solid line) and ischemic conditioned (open circles; dashed line) groups (pooled data). Note that cellular infarct size is greater in relation to increases in myocardial oxyen demand (i.e. non conditioned: $\mathrm{Y}=37.6+6.74 \mathrm{x} ; \mathrm{r}^{2}=0.0 .89$ vs. ischemic conditioned: $\mathrm{Y}=46.3-2.04 \mathrm{x} ; \mathrm{r}^{2}=0.85$ ); no statistical differences were obtained between the treatment groups.

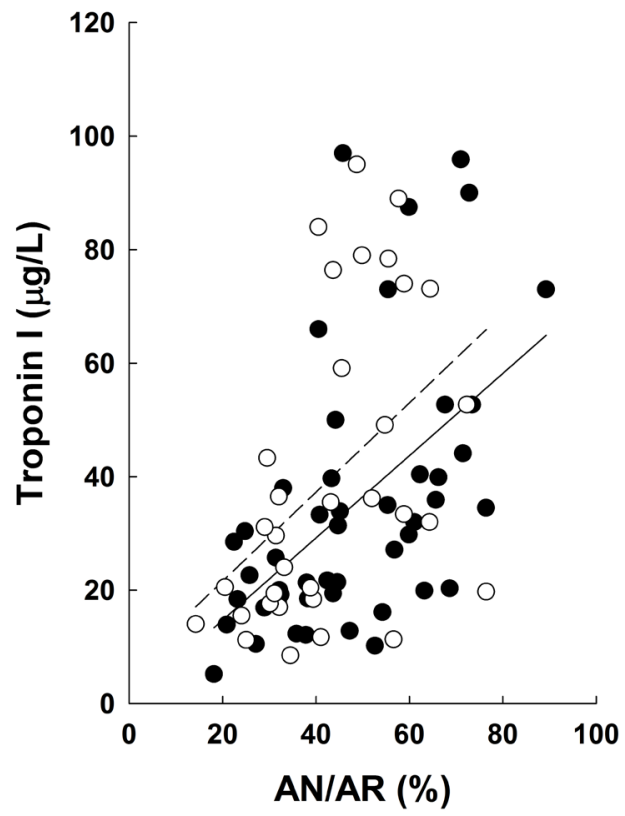

Figure 4. Scattergram showing the relation between infarct size (as percent of risk zone size) and serum troponin I levels for non-conditioned (closed circles; solid line) and ischemic conditioned (open circles; dashed line) groups (pooled data). Note the higher serum troponin I levels in relation to increased cellular injury (i.e. non conditioned: $\mathrm{Y}=$ $0.22+0.72 \mathrm{x} ; \mathrm{r}^{2}=0.29$ vs. ischemic conditioned: $\left.\mathrm{Y}=5.86+0.79 \mathrm{x} ; \mathrm{r}^{2}=0.20\right)$; despite the trend to smaller infarcts with ischemic conditioning serum troponin I levels were not substantially lower. 


\section{Discussion}

Determining whether ischemic conditioning can delay development of cellular injury caused by vascular obstruction for prolonged periods has significant clinical implications. For instance, in patients with ongoing myocardial infarction reperfusion therapy is often delayed for extended periods; whether ischemic conditioning can help to reduce injury until such time that reperfusion therapy can be instituted remains unanswered. The results of the present study substantiate numerous earlier studies that treatment by ischemic conditioning prior to a prolonged ischemic event delays progression of cellular necrosis within the risk zone of the infarct-related artery; however, the protection afforded by ischemic conditioning is finite in that with prolonged ischemia protection is lost. Our findings indicate that protection, or delayed development of ischemic injury, only occurs with brief ischemic periods (i.e. $<60-\mathrm{min}$ ). Interestingly, we noted a trend to smaller infarcts (not significant) in ischemic conditioned rabbits subject to longer periods of coronary occlusion. No protection was obtained in ischemic conditioned rabbits undergoing 360-min coronary occlusion. These findings support the hypothesis that ischemic conditioning delays progression of cellular injury; however, protection is highly dependent on duration of ischemia.

In most in vivo studies with rabbits the duration of coronary occlusion is 30-, or 45-min; within this period ischemic conditioning exerts a marked protective response by reducing infarct size by 35 - 50 percent [21]. In the present study, infarct size was reduced by 35 percent compared to non-conditioned rabbits. Significant reduction of tissue necrosis in rabbits subject to ischemic conditioning ( 2 cycles occlusion/reperfusion) with 120-min coronary occlusion has been reported [21]; interestingly, findings from the same laboratory have also reported that ischemic conditioning failed to limit necrosis in rabbits subject to the same duration of coronary occlusion [22]. The major difference between the two studies was the duration of ischemia within the ischemic conditioning protocol. As such, the duration of ischemia appears to play a role in stimulating the conditioning response at the cellular level.

Mechanisms responsible for stimulating the anti-necrosis properties of ischemic conditioning during vascular occlusion (heart and other organs) have been discussed elsewhere [2] [23]. For the early ischemic conditioning phenomenon, putative mechanisms include: recruitment of coronary collateral vessels, induction of reactive oxygen intermediate scavengers, synthesis of protective proteins and stimulation of numerous cell receptors [24]. For delayed ischemic conditioning alterations in protein synthesis (i.e. not only protein activity) within the conditioned myocardium appears to be the predominant contributory mechanism [25]. Identification of cellular triggers, transducers and end-effectors activated by ischemic conditioning has been the focus of abundant investigations over the past several decades.

Various serologic biomarkers (cardiac troponins, creatine kinase, lactate dehydrogenase, myoglobin) are used to confirm diagnosis of myocardial infarction 
[26] [27] [28]; however, the accuracy of these determinations is often questioned. With regard to physiopathology, troponin release from myofibrils varies depending on the extent of myocyte injury; other important factors that affect overall efficacy of troponin assessment include the level of reperfusion and the extent of inflammation within the infarct area. In patients, serum troponins are mostly used as a surrogate endpoint for infarct size assessment [29]. Cardiac troponin I is the inhibitory subunit of troponin; this isoform is highly cardiac specific and is not found in skeletal muscle [30]. Pharmacologic interventions can reduce serum levels of troponins in addition to their cytoprotective actions [31]; however, this has not been widely studied. In the present study, we examined the influence of ischemic conditioning on release of troponin I (i.e. gold standard) following extended ischemia. Our findings show a good correlation between myocardial infarction and serum troponin I levels as expected; serum troponin I levels increased substantially with greater myocardial injury but no differences between these correlations was observed between non-conditioned and ischemic conditioned groups. In the only group with documented cardioprotection by ischemic conditioning (i.e. 30-min coronary occlusion) statistical differences for serum troponin I levels were not observed $(23.3 \pm 10.5$ vs $16.0 \pm 7.5 \mu \mathrm{g} / \mathrm{L}$, nonconditioned vs. ischemic conditioned; mean $\pm 1 \mathrm{SD}, \mathrm{p}=0.116$ ).

This study has some limitations. First, we used an ischemic conditioning stimulus of 2 cycles of 5-min coronary occlusion and 5-min coronary reperfusion; typically, one to four 5-min ischemic periods have been used to stimulate protection in the heart in rabbits. Shorter [32] [33], or longer [34] durations of ischemia within the conditioning protocol do not afford additional benefit; the triggering stimulus is mostly created by the abrupt interruption in blood flow which evidently stops oxygen and nutrient delivery. Coronary collateral blood flow which is an important determinant of infarct size [19] [35] was not evaluated in the present study as it has been documented to be mostly non-existent in this specific animal model [36] [37]. However, Hale and co-workers evaluated coronary collateral blood flow during reperfusion in ischemic conditioned rabbits in several studies and showed improved blood flow within the area at risk [21] [38]; improved post-ischemic blood flow is probably a result of greater protection of coronary vessels within the risk area due to ischemic conditioning.

\section{Conclusion}

Ischemic conditioning delays development of myocardial injury; however, protection is reserved for relatively short durations of ischemia and is not preserved with acute coronary occlusions greater than 4 hours. It is clear from our findings that ischemic conditioning can protect ( 20 percent) myocardium even with coronary occlusions that last up to 4 hours. Whether this is important for patients with acute myocardial infarction of prolonged duration remains to be determined; however, it is clear that some protection against cell death is better than none. Hemodynamic and biomarker findings observed in this study con- 
firm previously published results from earlier studies; troponin I levels increased in relation to infarct size as expected. Further studies are needed to determine physiopathological mechanisms and therapeutic strategies to limit cellular injury resulting from acute and chronic coronary occlusion.

\section{Acknowledgements}

This study was supported, in part, by a grant from the Heart and Stroke Foundation of Québec. We thank Dr. Phung (recipient of a Canadian Association for International Development Scholarship) for his help in carrying out the experiments.

\section{Conflicts of Interest}

The author declares no conflicts of interest regarding the publication of this paper.

\section{References}

[1] Murry, C.E., Jennings, R.B. and Reimer, K.A. (1986) Preconditioning with Ischemia: A Delay of Lethal Cell Injury in Ischemic Myocardium. Circulation, 74, 1124-1136. https://doi.org/10.1161/01.CIR.74.5.1124

[2] Hausenloy, D.J., Barrabes, J.A., Botker, H.E., Davidson, S.M., Di Lisa, F., Downey, J., et al. (2016) Ischaemic Conditioning and Targeting Reperfusion Injury: A 30 Year Voyage of Discovery. Basic Research in Cardiology, 111, 70. https://doi.org/10.1007/s00395-016-0588-8

[3] Heusch, G. (2013) Cardioprotection: Chances and Challenges of Its Translation to the Clinic. The Lancet, 381, 166-175. https://doi.org/10.1016/S0140-6736(12)60916-7

[4] Hausenloy, D.J., Kharbanda, R., Rahbek Schmidt, M., Moller, U.K., Ravkilde, J., Okkels, J.L., et al. (2015) Effect of Remote Ischaemic Conditioning on Clinical Outcomes in Patients Presenting with an ST-Segment Elevation Myocardial Infarction Undergoing Primary Percutaneous Coronary Intervention. European Heart Journal, 36, 1846-1848.

[5] Pickard, J.M., Botker, H.E., Crimi, G., Davidson, B., Davidson, S.M., Dutka, D., et al. (2015) Remote Ischemic Conditioning: From Experimental Observation to Clinical Application: Report from the 8th Biennial Hatter Cardiovascular Institute Workshop. Basic Research in Cardiology, 110, 453. https://doi.org/10.1007/s00395-014-0453-6

[6] Dawn, B. and Bolli, R. (2002) Role of Nitric Oxide in Myocardial Preconditioning. Annals of the New York Academy of Sciences, 962, 18-41. https://doi.org/10.1111/j.1749-6632.2002.tb04053.x

[7] Goto, M., Liu, Y., Yang, X.-M., Ardell, J.L., Cohen, M.V. and Downey, J.M. (1995) Role of Bradykinin in Protection of Ischemic Preconditioning in Rabbit Hearts. Circulation Research, 77, 611-621. https://doi.org/10.1161/01.RES.77.3.611

[8] Yellon, D.M. and Downey, J.M. (2003) Preconditioning the Myocardium: From Cellular Physiology to Clinical Cardiology. Physiological Reviews, 83, 1113-1151. https://doi.org/10.1152/physrev.00009.2003

[9] Murphy, E. and Steenbergen, C. (2008) Mechanisms Underlying Acute Protection from Cardiac Ischemia-Reperfusion Injury. Physiological Reviews, 88, 581-609. 
https://doi.org/10.1152/physrev.00024.2007

[10] Currie, R.W., Tanguay, R.M. and Kingma, J.G. (1993) Heat-Shock Response and Limitation of Tissue Necrosis during Occlusion/Reperfusion in Rabbit Hearts. Circulation, 87, 963-971. https://doi.org/10.1161/01.CIR.87.3.963

[11] Bolli, R. (2000) The Late Phase of Preconditioning. Circulation Research, 87, 972-983. https://doi.org/10.1161/01.RES.87.11.972

[12] Schaller, B. (2005) Ischemic Preconditioning as Induction of Ischemic Tolerance after Transient Ischemic Attacks in Human Brain: Its Clinical Relevance. Neuroscience Letters, 377, 206-211. https://doi.org/10.1016/j.neulet.2004.12.004

[13] Lee, H.T. and Emala, C.W. (2000) Protective Effects of Renal Ischemic Preconditioning and Adenosine Pretreatment: Role of A(1) and A(3) Receptors. American Journal of Physiology-Renal Physiology, 278, F380-F387. https://doi.org/10.1152/ajprenal.2000.278.3.F380

[14] Schmidt, M.R., Pryds, K. and Botker, H.E. (2014) Novel Adjunctive Treatments of Myocardial Infarction. World Journal of Cardiology, 6, 434-443.

https://doi.org/10.4330/wjc.v6.i6.434

[15] Hausenloy, D.J., Mwamure, P.K., Venugopal, V., Harris, J., Barnard, M., Grundy, E., et al. (2007) Effect of Remote Ischaemic Preconditioning on Myocardial Injury in Patients Undergoing Coronary Artery Bypass Graft Surgery: A Randomised Controlled Trial. The Lancet, 370, 575-579. https://doi.org/10.1016/S0140-6736(07)61296-3

[16] Brevoord, D., Kranke, P., Kuijpers, M., Weber, N., Hollmann, M. and Preckel, B. (2012) Remote Ischemic Conditioning to Protect against Ischemia-Reperfusion Injury: A Systematic Review and Meta-Analysis. PLoS ONE, 7, e42179. https://doi.org/10.1371/journal.pone.0042179

[17] Libersan, D., Khalil, A., Quan, E., Kallaayoune, R., Tran, D. and Latour, J.-G. (1993) The Low-Molecular Weight Heparin: Enoxaparin Reduces Infarct Size When Given at Reperfusion in Untreated and Streptokinase-Treated Dogs. Journal of Molecular and Cellular Cardiology, 25, S90.

[18] Vitola, J.V., Morrow, J.A., Ingram, D.A., Murray, J.J. and Forman, M.B. (1993) Lidocaine Reduces Infarct Size in the Rabbit Model of Myocardial Reperfusion Injury and Inhibits Neutrophil Activation in Vitro. Circulation, 88, I545.

[19] Reimer, K.A., Jennings, R.B., Cobb, F.R., Murdock, R.H., Greenfield, J.C., Becker, L.C., et al. (1985) Animal Models for Protecting Ischemic Myocardium (AMPIM): Results of the NHLBI Cooperative Study. Comparison of the Unconscious and Conscious Dog Models. Circulation Research, 56, 651-665.

https://doi.org/10.1161/01.RES.56.5.651

[20] Ytrehus, K., Liu, Y., Tsuchida, A., Miura, T., Liu, G.S., Yang, X.-M., et al. (1994) Rat and Rabbit Heart Infarction: Effects of Anesthesia, Perfusate, Risk Zone, and Method of Infarct Sizing. American Journal of Physiology-Heart and Circulatory Physiology, 267, H2383-H2390. https://doi.org/10.1152/ajpheart.1994.267.6.H2383

[21] Hale, S.L. and Kloner, R.A. (1999) Ischemic Preconditioning and Myocardial Hypothermia in Rabbits with Prolonged Coronary Artery Occlusion. American Journal of Physiology, 276, H2029-H2034. https://doi.org/10.1152/ajpheart.1999.276.6.H2029

[22] Reffelmann, T., Hale, S.L., Li, G. and Kloner, R.A. (2002) Relationship between No Reflow and Infarct Size as Influenced by the Duration of Ischemia and Reperfusion. American Journal of Physiology-Heart and Circulatory Physiology, 282, H766-H772. https://doi.org/10.1152/ajpheart.00767.2001 
[23] Kingma, J.G. (2014) Conditioning Strategies Limit Cellular Injury? World Journal of Cardiovascular Diseases, 4, 539-547. https://doi.org/10.4236/wjcd.2014.411065

[24] Eisen, A., Fisman, E.Z., Rubenfire, M., Freimark, D., McKechnie, R., Tenenbaum, A., et al. (2004) Ischemic Preconditioning: Nearly Two Decades of Research. A Comprehensive Review. Atherosclerosis, 172, 201-210.

https://doi.org/10.1016/S0021-9150(03)00238-7

[25] Rizvi, A., Tang, X.L., Qiu, Y., Xuan, Y.T., Takano, H., Jadoon, A.K., et al. (1999) Increased Protein Synthesis Is Necessary for the Development of Late Preconditioning against Myocardial Stunning. American Journal of Physiology, 277, H874-H884. https://doi.org/10.1152/ajpheart.1999.277.3.H874

[26] Steenbergen, C., Perlman, M.E., London, R.E. and Murphy, E. (1993) Mechanism of Preconditioning. Ionic Alteration. Circulation Research, 72, 112-125. https://doi.org/10.1161/01.RES.72.1.112

[27] Omar, B.A., Hanson, A.K., Bose, S.K. and McCord, J.M. (1991) Ischemic Preconditioning Is Not Mediated by Free Radicals in the Isolated Rabbit Heart. Free Radical Biology and Medicine, 11, 517-520. https://doi.org/10.1016/0891-5849(91)90064-A

[28] Yamahara, Y., Asayama, J., Kobara, M., Ohta, B., Matsumoto, T., Miyazaki, H., et al. (1994) Effects of Ischemic Preconditioning on the Release of Cardiac Troponin T in Isolated Rat Hearts. Basic Research in Cardiology, 89, 241-249.

[29] Schaaf, M., Huet, F., Akodad, M., Gorce-Dupuy, A.M., Adda, J., Macia, J.C., et al. (2019) Which High-Sensitivity Troponin Variable Best Characterizes Infarct Size and Microvascular Obstruction? Archives of Cardiovascular Diseases, 112, 334-342. https://doi.org/10.1016/j.acvd.2018.12.001

[30] Vallins, W.J., Brand, N.J., Dabhade, N., Butler-Browne, G., Yacoub, M.H. and Barton, P.J. (1990) Molecular Cloning of Human Cardiac Troponin I Using Polymerase Chain Reaction. FEBS Letters, 270, 57-61. https://doi.org/10.1016/0014-5793(90)81234-F

[31] Letienne, R., Bel, L., Bessac, A.M., Denais, D., Degryse, A.D., John, G.W., et al. (2006) Cardioprotection of Cariporide Evaluated by Plasma Myoglobin and Troponin I in Myocardial Infarction in Pigs. Fundamental \& Clinical Pharmacology, 20, 105-113. https://doi.org/10.1111/j.1472-8206.2006.00394.x

[32] Van Winkle, D.M., Thornton, J., Downey, D.M. and Downey, J.M. (1991) The Natural History of Preconditioning Cardioprotection Depends on Duration of Transient Ischemia and Time to Subsequent Ischemia. Coronary Artery Disease, 2, 613 619.

[33] Miura, T. and Iimura, O. (1993) Infarct Size Limitation by Preconditioning: Its Phenomenological Features and the Key Role of Adenosine. Cardiovascular Research, 27, 36-42. https://doi.org/10.1093/cvr/27.1.36

[34] Yamasaki, K., Fujiwara, H., Tanaka, M., Yokota, R., Miyamae, M., Ogawa, J., et al. (1997) Preconditioning with 15-Minute Ischemia Extends Myocardial Infarct Size after Subsequent 30-Minute Ischemia in Rabbits. Japanese Circulation Journal, 61, 344-352. https://doi.org/10.1253/jcj.61.344

[35] Neinaber, C., Mauser, M. and Schaper, W. (1983) Stimulation of Myocardial Adenine Nucleotide Synthesis by Postischemic Reperfusion with Direct Purine Precursor AICAR ${ }^{\star}$-Comparison with Ribose $\left({ }^{\star} 5\right.$-Amino-4-imidazole-carboxamide-riboside). Journal of the American College of Cardiology, 1, 667.

[36] Maxwell, M.P. (1994) How to Identify and Quantify an Underperfused Zone. Cardiovascular Research, 28, 570-573. https://doi.org/10.1093/cvr/28.4.570

[37] Maxwell, M.P., Hearse, D.J. and Yellon, D.M. (1987) Species Variation in the Coro- 
nary Collateral Circulation during Regional Myocardial Ischemia: A Critical Determinant of the Rate of Evolution and Extent of Myocardial Infarction. Cardiovascular Research, 21, 737-746. https://doi.org/10.1093/cvr/21.10.737

[38] Hale, S.L. and Kloner, R.A. (1992) Effect of Ischemic Preconditioning on Regional Myocardial Flow in the Rabbit Heart. Coronary Artery Disease, 3, 133-140.

https://doi.org/10.1097/00019501-199202000-00008 